\title{
CREATING A MODEL MODULE FOR THE NOVEL RESOURCE DMU E-PARASITOLOGY
}

\author{
A. Peña-Fernández ${ }^{1}$, M.D. Ollero ${ }^{2}$, S. Fenoy ${ }^{2}$, A. Magnet ${ }^{2}$, F. Izquierdo ${ }^{2}$, \\ M.Á. Peña ${ }^{3}$, FJ. Bornay ${ }^{4}$, L. Acosta ${ }^{4}$, L.A. Parker ${ }^{4}$, T. Sgamma ${ }^{1}$, C. del Águila ${ }^{2}$ \\ ${ }^{1}$ School of Allied Health Sciences, De Montfort University (UNITED KINGDOM) \\ ${ }^{2}$ Facultad de Farmacia, Universidad San Pablo CEU (SPAIN) \\ ${ }^{3}$ Universidad de Alcalá, Departamento de Ciencias Biomédicas (SPAIN) \\ ${ }^{4}$ Universidad Miguel Hernández de Elche (SPAIN)
}

\begin{abstract}
The study of parasitology has become essential to build future health care professionals with skills to respond to public health threats such as the recent outbreaks due to Cryptosporidium spp. or Giardia in the United Kingdom (UK). To facilitate the teaching of parasitology, which negligible across the different undergraduate and taught masters degrees at De Montfort University (DMU, Leicester, UK), a group from different EU Universities [DMU and the Spanish universities: University of San Pablo CEU (USP-CEU) and University Miguel Hernández], clinicians and practising Biomedical Scientists from the UK National Health Service are developing an on-line package for teaching and learning parasitology named DMU e-Parasitology. This package will have three sections or modules: a theoretical module with mini e-learning units to study major human parasites such as Leishmania spp. or malaria; a virtual laboratory module with units to enhance the learning and study of parasitic diseases such as culture of parasites, staining and use of light microscope to identify these organisms or molecular techniques for the identification of parasites to species level; and a virtual microscope with a real slide collection of clinical samples of parasites. The DMU e-Parasitology resource is being created for undergraduate/postgraduate human health science students, with corresponding degrees of difficulty. Units will include a tool to assess the learning process of the students, in form of a quiz, activity and/or exam, and several formative activities throughout each mini-module. The development of this teaching resource will cover a gap in the traditional teaching and learning methods that are currently used and provided in the participating universities. The DMU e-Parasitology will help our undergraduate students to gain significant knowledge in parasitology by promoting self-learning. A unit related to Toxocara, a helminthiasis with prevalence rates that can reach as high as $40 \%$ or more in different parts of the world, was firstly developed to use as a model for the development of the DMU eParasitology. Three undergraduate students that studied parasitology during the first term in 2016/17 [6 European Credit Transfer and Accumulation System credits (ECTS); $3^{\text {rd }}$ year module] from the bilingual Pharmacy and Biotechnology degree at USP-CEU were voluntarily recruited to provide comprehensive feedback for this model unit at the beginning of the second term. This unit was tested with these students because of their comprehensive knowledge of parasitology. Students described the model unit as interactive and presenting the appropriate content and resources to study the parasitic disease addressed (toxocariasis). Limitations were the poor navigability in the formative exercise section and the excessive information provided in some slides that could hinder their understanding. The team has addressed these limitations and is using this unit as a model to build the DMU e-Parasitology, which will be accessible through the DMU website (http://parasitology. dmu.ac.uk) in 2018. We consider that this teaching and learning resource will overcome barriers of time, space, equipment and resources; and may help students and scientists around the world in the diagnosis of different parasitic diseases that impact human health.
\end{abstract}

Keywords: DMU e-Parasitology, teaching and learning parasitology, e-Learning.

\section{INTRODUCTION}

The use of modern technology in medical and biomedical sciences such as the use of virtual environments and eLearning packages can enhance students understanding, engagement and provide time and space flexibility to learn $[1,2]$. In addition, the use of web-based teaching resources is an increasingly important method for delivering education and it will be particularly important in the near future due to the progressively increasing number of health science students in conjunction with the current limited number of academics in the "European Higher Education Area", and to facilitate the process and management of the increase in medical and biomedical knowledge. However, different 
authors have described limitations to eLearning particularly related to practical and/or technical factors such as difficulties accessing websites, quality of the final product and/or little support provided [2-4]. Reid et al. (2016) [1] have highlighted that the user experience with eLearning is not completely described, hence the relevance of studies to explore the obstacles that learners have to engage with eLearning.

The study of parasitology and infectious diseases is essential to build professionals in the health sector with the key knowledge and skills to face global public health threats such as food-, water- or vector-borne infectious diseases outbreaks. Examples of recent outbreaks in the UK that have highlighted the necessity of highly skilled health professionals to protect the public health are related to Cryptosporidium spp. [5]. However, the current time dedicated to the teaching of these disciplines in all health sciences degrees at De Montfort University (DMU, Leicester, UK) is minimal or non-existent depending on the degree programme. Therefore, an innovative teaching group from different EU Universities [DMU and the Spanish universities: University of San Pablo CEU (USP-CEU) and University Miguel Hernández], clinicians and practising Biomedical Scientists from the UK National Health Service are developing a complete on-line package for teaching and learning parasitology named DMU e-Parasitology which will also cover topics related to infectious diseases. The DMU eParasitology will focus on parasites that affect humans.

\section{METHODOLOGY}

The aims of this study were to describe the development of the unit that will be used as a model to build the future DMU e-Parasitology and the methods used to develop an appropriate and robust model-unit. To do this, a focus group was created with volunteer students to gather preliminary information about this first model-unit. A feedback questionnaire was distributed within this group to collect comprehensive feedback to address their comments, develop the model-unit and investigate if the comprehensive curriculum modifications performed in the BMS programme at DMU have been translated in an improvement in the knowledge of parasitology and infectious diseases in our BMS students.

\subsection{DMU e-Parasitology}

The novel DMU e-Parasitology package will be publicly available at the DMU website here http://parasitology.dmu.ac.uk/ in 2018. Its development started in 2016 and it is being created for undergraduates and postgraduates studying any human health science programme, so different degrees of difficulty are being considered and targeted. Two DMU developers and different artists are collaborating with academics, parasitologists and biomedical scientists registered by the HCPC (UK Health and Care Professions Council) at the above EU academic arenas in the development of this novel eLearning package. The HCPC is the UK regulatory body that certifies that health and care professionals such as biomedical scientists meet training, professional skills, behaviour and health standards to deliver their professions [6]. Although a common perception may be that artists could have difficulty communicating science, they could enhance and facilitate the communication of science when working with scientists. Artists can create an impact on society (and therefore students) through using different resources such as visually impacting images or artwork. Artworks can facilitate understanding and enhance knowledge transfer/retention, facilitate student engagement, visual model-based reasoning and simplify the communication of complex concepts.

\subsubsection{Sections of the DMU e-Parasitology}

The DMU e-Parasitology is being created using the Articulate 360 software and will have three sections or modules (Fig. 1):

- A theoretical module with mini e-learning units to study major human parasites such as Leishmania spp. or malaria. This module will cover the three main classes of parasites that can affect humans, i.e. protozoa, helminths and ectoparasites. To facilitate their study and identification, the different parasites have been grouped in headings and the three common taxa considered for helminths, i.e. trematodes, cestodes and nematodes are also considered (Fig. 2). Finally, owing to their impact on human morbidity and public health, the group of fungal parasites microsporidia will be also addressed. 
(2)

\section{eParasitology}

Learning Resources for the Study of Parasites

Home

This website is currently under development.

e-Learning Units and Virtual Microscope

leam about a number of parasites. You

accessing our virtual microscope to look at parasites in great detail
High Magnification Images and Laboratory Tools/Skills

You will have the opportunity to look at highly magnified images. Also you will learn about the technical tools and skills that you will need when working in a laboratory

Figure 1. Overview of the main page of the future DMU e-Parasitology (Image courtesy of DMU; PeñaFernández et al., 2017). Available at: http://parasitology.dmu.ac.uk/index.htm

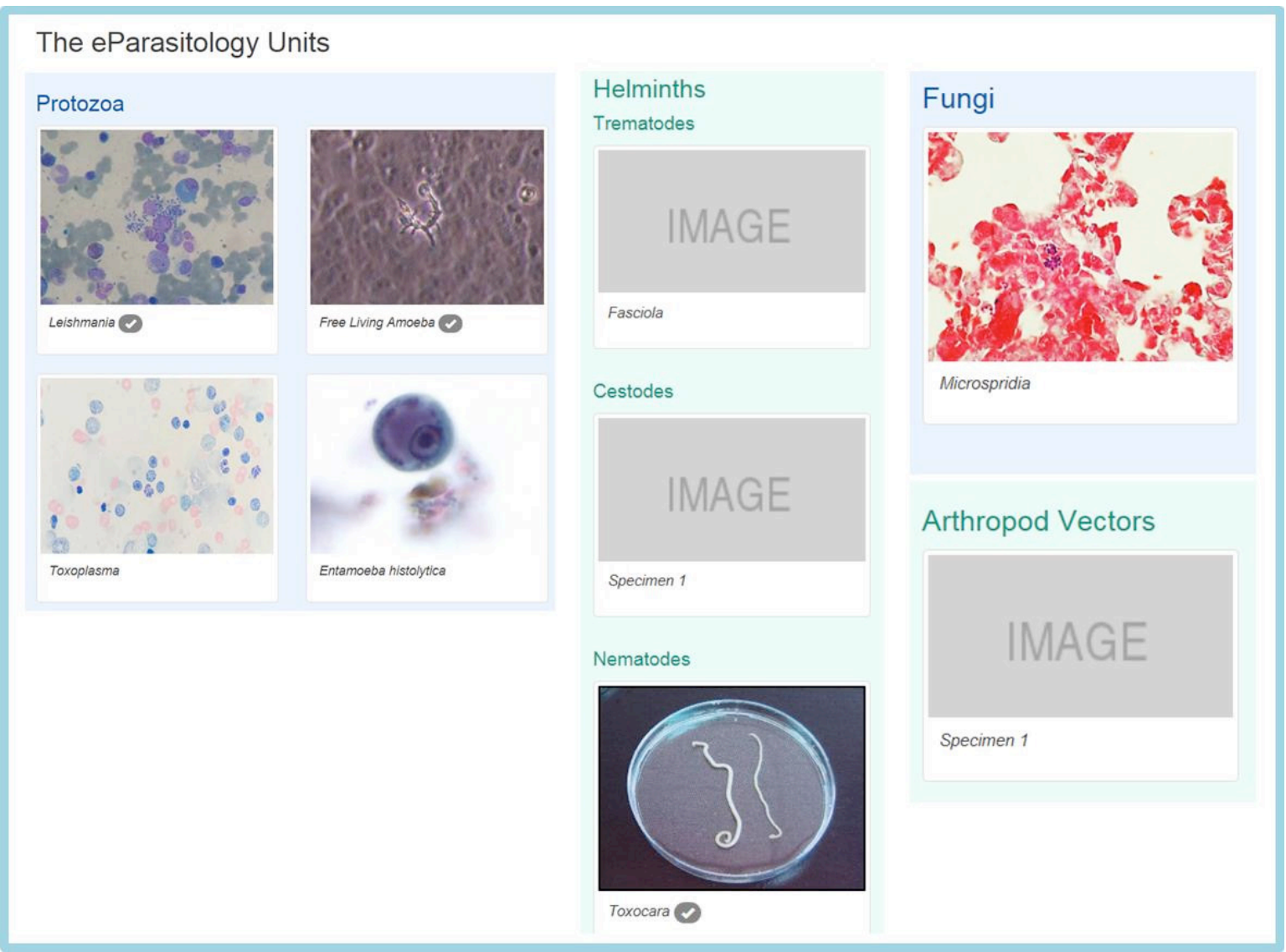

(Image for Leishmania is courtesy of Paulo H. Orlandi, 2009 (CC-BY-SA 3.0), available at https://commons. wikimedia. org/wiki/File:Leishmania_2009-04-14_smear.JPG; photo for Entamoeba is from the Image Gallery of the CDC website https://www.cdc.gov/dpdx/amebiasis/index.html; the rest of the images are courtesy of Laboratorio de Parasitología at the USP-CEU)

Figure 2. Overview of the theoretical module of the DMU e-Parasitology.

- A virtual laboratory module with engaging and interactive units about different techniques which are relevant for biomedical sciences, including parasitology, is being developed to enhance the learning and study of parasitic diseases. These units include a description of different equipment and videos of academics working with them to facilitate the understanding and 
enhance student experience. Key units in this section are related with cell and parasites culture, routine techniques for identifying these organisms (e.g. staining techniques) in any clinical samples, or molecular techniques to determine their species including Polymerase Chain Reaction. In addition, we are creating units about general equipment in any biomedical science laboratory such as related with microscopes, pipettes and more specifically such as the class II biological safety cabinet.

- A virtual microscope with a real slide collection of clinical samples of human parasites. Ordinary micrographs or photographs are limited to a preselected area and magnification but the virtual microscopy will enable viewing of any part of a specimen at any magnification and, therefore, students will be able to identify morphologically the parasite in different clinical samples.

\subsubsection{Development of units}

Academics, parasitologists and biomedical scientists collaborating in this teaching project are completing a proforma that will be used for developing the on-line units. To complete the proforma, collaborators will perform a comprehensive literature review and will collect the most current information for each disease for the following main points: a) infectious agent, life-cycle and transmission, b) risk factors and epidemiology, c) clinical and pathologic features, d) diagnosis, e) treatment and prevention. The units for the practical section will not follow a scheme or a given structure.

All units in both modules in the DMU e-Parasitology will include some formative assessments so the user of this package will be able to assess their learning process throughout the unit.

\subsubsection{Development of the model-unit and focus group screening}

The first unit developed to use as a model was related with the helminth Toxocara, as present prevalence rates that can reach as high as $40 \%$ or more in different parts of the world. We followed the above methods to develop this unit (Fig. 3).

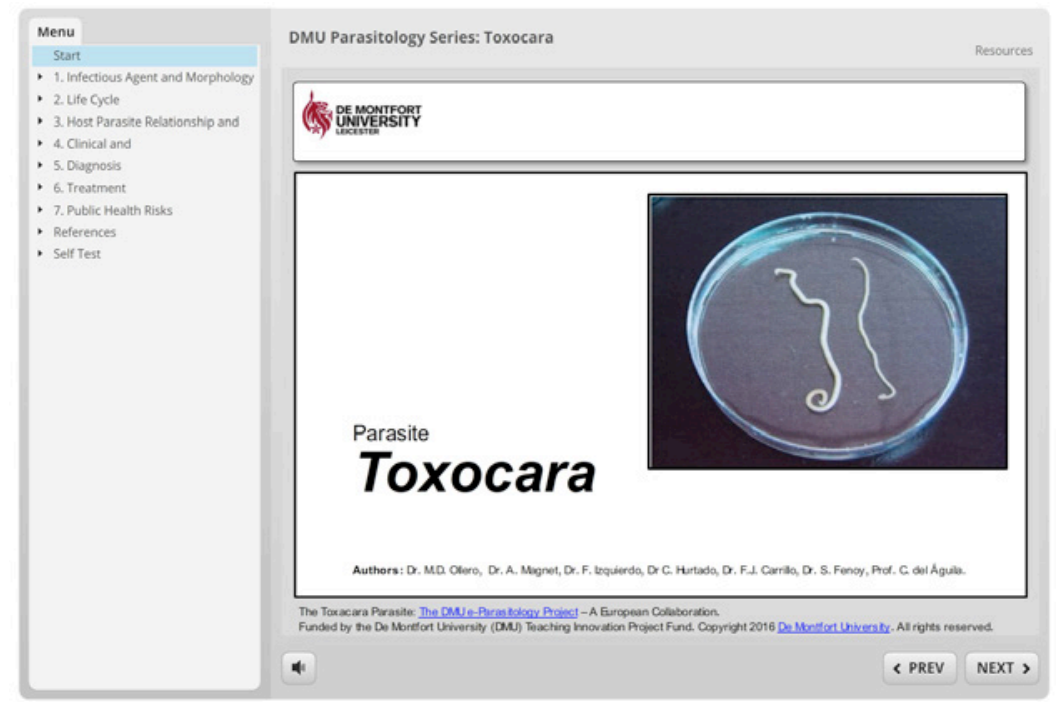

Figure 3. Overview of the main page of the unit Toxocara in the DMU e-Parasitology (Image courtesy of DMU; Peña-Fernández et al., 2017). Available at: http://parasitology.dmu.ac.uk/learn/modules/toxocara/story.html

The focus group was recruited from undergraduate students from the bilingual Pharmacy and Biotechnology degree at USP-CEU that studied parasitology during the first term during 2016/17 [6 European Credit Transfer and Accumulation System credits (ECTS); 3rd year module; 27 students were enrolled in this module] [7]. This cohort of students was identified as one of the most knowledgeable in parasitology within the overseas participating universities during this course. Only 3 students voluntarily provided comprehensive feedback for this model-unit at the beginning of the second term. A link to the Toxocara unit was provided to these students and, after testing it, completed an anonymous feedback questionnaire with some open-answer questions related to what they had found useful about this unit and areas or items to change or improve. 


\section{RESULTS \& DISCUSSION}

Students highlighted a high degree of satisfaction with the Toxocara unit in the surveys (100\% agreed) and enjoyed the experience with the DMU e-Parasitology (66.7\% agreed; $33.3 \%$ strongly agreed). In addition, they have described that the module is highly interactive, easy to understand, and with exercises relevant to the study of the Toxocara parasite.

In relation with the usefulness of the unit and the website, some students have commented that "it was an easy-to-use webpage", a useful tool as "saving-time method to search for correct information" or as a "good idea to complement the subject" or to clarify information that was unclear during the lecture when studying. Students have also indicated that the unit particularly helped them to have a better understanding of the life-cycle of Toxocara.

Elements to improve the unit and the DMU e-Parasitology website pointed out by the focus group were related with the technical part of the package, such as improving the navigation during the exercises, or reducing the information of some slides to facilitate their assimilation and understanding.

The academic team involved in the development of this resource also provide some feedback to the developers about the unit and the main site, which resulted in the completion of the model-unit that is being followed to develop the rest of the units. The major challenge that we have found to develop the DMU e-Parasitology so far are related with resources, both staffing and technical, as developing an appropriate eLearning resource for different health science students is challenging due to the different backgrounds.

The present study only focuses on a limited number of students and their perceptions, hence further studies are needed to determine whether the DMU e-Parasitology would be an appropriate tool for learning parasitology. Thus, this resource will be tested during the course 2017/18 at the three participating universities to collect comprehensive feedback that will not only be used for analysing its utility and efficacy but also to complete appropriately the e-Parasitology project by the end of 2018 . This is a key part of this teaching project as it will permit students to be a producer and to be involved in the creation of this package. Finally, the effectiveness of the virtual microscope on learning will be assessed by creation of mini case-studies in which students will be asked to identify the parasites in the virtual slides displayed during different workshops in each academic arena.

\section{CONCLUSIONS}

Students enjoyed the drafted unit and provided comprehensive feedback to complete it appropriately. This unit on Toxocara is being used as a model to develop the future DMU e-Parasitology, which will be tested during the course 2017/18. Students have pointed out that the e-learning that we are creating can help with the study and understanding of the different human parasitic diseases. Additionally, the design of the life cycle was specially pointed out from students as really useful in the understanding of toxocariasis. Different artists will collaborate in this project, as they are a critical component of developing e-learning resources.

The DMU e-Parasitology will cover a gap in the traditional teaching and learning methods that are currently used and provided in the participating Universities. This teaching resource will aid to our undergraduate students to gain a significant knowledge in parasitology by promoting self-learning and overcoming barriers of time, space, equipment and resources.

\section{ACKNOWLEDGEMENTS}

The authors would like to express their sincere appreciation to Steve Mackenzie, Jonathan Coope and Maxine Armstrong (DMU) for their work to develop the DMU e-Parasitology and to Dr FJ. Carrillo for his life-cycle designs. Finally, we would like to thank the Teaching Innovation Project Fund at De Montfort University (scheme 2015-16) to fund this project to Dr. Peña-Fernández.

\section{REFERENCES}

[1] Reid HJ, Thomson C, McGlade KJ. Content and discontent: a qualitative exploration of obstacles to elearning engagement in medical students. BMC Med Educ 2016; 16:188. 
[2] Polly P, Marcus N, Maguire D, Belinson Z, Velan GM. Evaluation of an adaptive virtual laboratory environment using Western Blotting for diagnosis of disease. BMC Med Educ 2014; 14:222.

[3] Docherty A, Sandhu H. Student-perceived barriers and facilitators to e-learning in continuing professional development in primary care. Educ Prim Care 2006; 17(4):343-353.

[4] Moule P, Ward R, Lockyer L. Nursing and healthcare students' experiences and use of elearning in higher education. J Adv Nurs 2010; 66:2785-2795.

[5] McKerr C, Adak GK, Nichols G, Gorton R, Chalmers RM, Kafatos G, Cosford P, et al. An Outbreak of Cryptosporidium parvum across England \& Scotland Associated with Consumption of Fresh Pre-Cut Salad Leaves, May 2012. PLoS One 2015; 10(5):e0125955.

[6] HCPC website. Available at: http://www.hcpc-uk.co.uk/aboutus/ [accessed 15/09/2017].

[7] Syllabus Parasitology module. Available at the USP-CEU website: http://www.uspceu.com/ _docs/oferta-academica/fac_far/farmacia/guias-docentes/GD5-a301-Parasitology.pdf [accessed 28/09/2017]. 